\title{
The Use of GPR in Delineating an Iron Sand Boundary and the Determination of Its Electromagnetic Wave Velocity: A Case Study in Jepara, Central Java
}

\author{
S. Bijaksana ${ }^{1}$ I. Rusyanti ${ }^{2}$, M. I. T. Taib ${ }^{2}$, L. A. Pasasa ${ }^{1}$ \& A. S. Andreas ${ }^{1}$ \\ ${ }^{1}$ Physics Department, Institut Teknologi Bandung, Jalan Ganesa 10 \\ Bandung 40132, Indonesia \\ ${ }^{2}$ Geophysical Engineering Department, Institut Teknologi Bandung, Jalan Ganesa 10 \\ Bandung 40132, Indonesia
}

\begin{abstract}
Exploring the vertical extent of iron sand deposit is challenging as conventional geophysical methods (electrical resistivity, geomagnetic, and seismic refraction) are inappropriate and unsuccessful in delineating the iron sand deposit from the bedrock. Ground-penetrating radar (GPR) offers a solution to the above problem as radar is not affected negatively by the physical properties of iron sand. In the year 2003, a RAMAC's GPR survey was carried out in the coast of Bayuran in the Regency of Jepara, Central Java to map the distribution of sub-bottom iron sand. The sand is highly magnetic. The survey used $100 \mathrm{MHz}$ antennas. The survey is also complimented by a novel method in determining the electromagnetic (EM) wave velocity of iron sand. Combination of reflection profiling and CMP sounding was deployed. Results of CMP sounding were processed using CMP-semblance analysis that produces the RMS velocity in velocity-time spectra. The RMS velocity is then converted to interval velocity using Dix's formula and is found to be about $135 \mathrm{~mm} / \mathrm{ns}$. Meanwhile, combination of magnetic susceptibility, relative permittivity, and dissipation factors produces radiowaves velocity of iron sand as a function of frequency. The velocities of radiowaves estimated from laboratory match that estimated from CMP analysis.
\end{abstract}

Keywords: Bayuran; EM velocity; GPR; iron sand; magnetic minerals.

\section{Introduction}

In Indonesian archipelago, especially in the Island of Java, there are huge deposits of iron sand originating from volcanic processes. Although these deposits have high content of iron and iron oxides, they were exploited only as raw material either as a mixture in cement production or simply as building material. These types of exploitation have low economic value.

With its high content of iron and iron oxides, iron sand could be turned, in fact, into more valuable industrial commodity [1]. Steel production has been tried since colonial time as a logical use of iron sand. The sand was processed and 
formed into pellets in steel production. Unfortunately, this kind of steel production is less economical than other kinds of steel production and therefore it has never been established as feasible industry.

However, recently, there is growing interest in exploiting the magnetic minerals in iron sand for more specific industrial materials. In a series of articles, the first author and his co-workers have publicized their research in iron sand. Magnetic characteristic of iron sand from the most notable deposit in Cilacap was published in 2002 [2], followed by a survey on magnetic characteristics of iron sands from eight different locations in Central Java [3]. Last paper on the series describes a method on producing industrial hematite $\left(\alpha \mathrm{Fe}_{2} \mathrm{O}_{3}\right)$ from iron sand [4].

Regardless of the actual use of iron sand, exploring the extent of iron sand deposit is quite challenging. While the acreage or the lateral extent of the deposit can be estimated easily, the thickness of the deposit varies greatly from one spot to another within the same locality. Based on earlier trial by the first author, conventional geophysical methods such as electrical resistivity, geomagnetic, and seismic refraction are inappropriate and unsuccessful in delineating the iron sand deposit from the bedrock. Electrical resistivity method fails as iron sand is highly conductive and is often soaked with conductive seawater. Similarly, geomagnetic method also fails, as iron sand is highly magnetic. Surveying iron sand deposit with seismic refraction method is also ineffective as the loose sand absorbs most of the seismic energy [5].

Ground penetrating radar (GPR) that became popular and readily available since the mid-1980s offers a solution to the above problem. Radar is not affected negatively by the physical properties of iron sand. With the advent of portable computers in the mid 1990s, GPR survey has become a practical and an effective approach in engineering and geophysical problems [6]. In recent years, the GPR method has been extended into refraction method [7] as well as into a full-resolution 3D imaging [8]. The responses of dispersive models have also been reported [9]. In a similar fashion, the importance of iron oxides, which is the major component of iron sand, as a cause of GPR reflection has also been reported [10].

In this paper, we report our survey as a maiden attempt to use GPR method in detecting the vertical extent of iron sand. The survey is carried out in coast of Bayuran in the Regency of Jepara, Central Java where the sand is highly magnetic compared to other iron sand locations in Java [3]. The survey is complimented by a novel method in determining the electromagnetic (EM) wave velocity of iron sand. The EM wave velocity produced by this method can be used as an alternative input for the conversion of travel time to actual depth 
that is crucial in GPR survey. The method could also be used to determine the EM wave velocity of other materials.

\section{Theory}

GPR method in a way is very similar to seismic method. The main difference between the two methods is in the types of waves that they use. While seismic method uses waves that consist of tiny packet of elastic strain energy, GPR uses electromagnetic waves in the form of radiowaves. A radar system comprises a signal generator, transmitting and receiving antennae, and a receiver. In some advanced systems, they are equipped with onboard computer that facilitates data processing while acquiring data in the field, and post-recording. The principles of GPR operation have been described in many geophysics textbooks [11-14].

The transmission antenna generates pulsed EM waves at certain frequency according to the characteristic of the antenna (between $10 \mathrm{MHz}$ to about 4 $\mathrm{GHz}$ ). The receiver antenna is then set to scan the received signal at the rate of 32-512 scans per second. This means that signal was transmitted every 2 to 31 $\mathrm{ms}$. Each scan is then displayed in the monitor as a function of two-way travel time, i.e., time required by the EM signal to travel from the transmitter to the target and then back to the receiver, normally given in the order of nano seconds. The display is called radargram. The ability of GPR to penetrate the ground depends on the frequency of the signal, the efficiency of radiating antenna, and the dielectric and magnetic properties of the materials. Depending on the signal bandwidth, higher frequency signals give higher vertical resolution but shallower depth of penetration and vice-versa. In textbooks [11-14], the range resolution, $\Delta R$, is approximately given by

$$
\Delta R=\frac{v}{2 \Delta f}
$$

where $\Delta f$ is the bandwidth of the transmitted spectrum and $v$ is the phase velocity of the medium.

Assuming that the interface between iron sand and the bedrock would act like a reflector, the first mode of radar deployment is a reflection profiling. In this mode, the antennae are moved simultaneously over the ground surface. Similar to seismic reflection profiling, the measured travel time to radar reflector is displayed on vertical axis while the distance the antenna has traveled is shown on the horizontal axis. The depth of the reflector can be determined if the radiowave velocities have been measured independently or known directly from borehole data. 
The determination of radiowave velocities is usually come from other modes of radar deployment, namely the wide-angle reflection and refraction (WARR) sounding and common midpoint (CMP) sounding. WARR sounding assumes that the material properties are uniform and that the reflector characteristics are the same over the area that the sounding is undertaken. The CMP is preferable, as it does not require such assumption. In CMP sounding, the transmitter and receiver are moved away from each other so that the midpoint between them stays at fixed location.

In analyzing the velocities, two statistical parameters namely the average velocity and the RMS velocity are used. The average velocity is the total ray path distance divided by total travel time, while the root-mean-square (RMS) velocity is the weighted-average velocity that applies to horizontal layers and normal incidence. The velocity of certain layer or interval can be calculated from RMS velocities using Dix's Formula [15]. The velocities are often determined with the aid of scaled semblance profiles. Semblance is a measure of the coherence of the CMP stacking process; when it equals 1 it implies perfect selection of the normal moveout correction. Coherence is a measure of the degree of fit of a theoretically derived hyperbolic curve at a given travel time for a chosen RMS velocity. With the aid of semblance profiles, it is easy to pick up the interval velocity through marking of reflection events.

Ideally, instead of in-situ determination through CMP sounding, the radiowave velocities could also be determined directly from the physical properties of the materials. In textbooks, such as [11], the velocity $\left(V_{m}\right)$ of a particular material is given by

$$
V_{m}=\frac{c}{\sqrt{\frac{\mu_{r} \varepsilon_{r}}{2}}\left(\sqrt{1+\frac{\sigma^{2}}{\omega^{2} \varepsilon^{2}}}+1\right)^{1 / 2}}
$$

where $c$ is the speed of light in free space $\left(3 \times 10^{8} \mathrm{~m} / \mathrm{s}\right), \mu_{r}$ is the relative magnetic permeability ( $=1$ for non-magnetic materials and is defined as $\mu_{r}=\mu$ / $\mu_{o}$, where $\mu$ is the magnetic permeability and $\mu_{o}$ is the magnetic permeability of free space), $\varepsilon$ is the permittivity $\left(=\varepsilon_{r} \varepsilon_{o}\right)$ and $\varepsilon_{o}$ is the permittivity of free space $\left(8.854 \times 10^{-12} \mathrm{~F} / \mathrm{m}\right), \varepsilon_{r}$ is the relative permittivity, $\sigma$ is the conductivity and $\omega=$ $2 \pi f$, where $f$ is the frequency.

From equation (2), one can calculate $V_{m}$ for a given material if $\mu_{r}, \varepsilon, \sigma$, and $\omega$ are known. The relative magnetic parameter $\mu_{r}$, can be calculated from the magnetic susceptibility $\chi$, measured easily by a magnetic susceptibility meter, through the following equation [11]. 


$$
\mu_{r}=\chi+1
$$

Permittivity, $\varepsilon$, meanwhile, is a complex quantity [13]. It is a measure of how much a medium change to absorb energy when subject to an electric field and is given by

$$
\varepsilon=\varepsilon^{\prime}+i \varepsilon^{\prime \prime}
$$

where $\mathbf{i}$ is a square root of $-1, \varepsilon$ 'and $\varepsilon "$ are the real and imaginary parts respectively [13]. The $\varepsilon$ " term is the frequency-dependent loss related to the relaxation response phenomena. In real materials, both real and imaginary parts of the permittivity are complicated functions of frequency. Therefore, appropriate determination of permittivity should be done as a function of frequency. Based on the Cole-Cole model on dispersion and absorption in dielectric, other relaxation models were developed including one that derived an equivalent circuit model [16], similar to a parallel RC circuit. In this model, the dielectric properties are determined from the measurement of impedance and admittance.

The relative permittivity $\varepsilon_{r}$ is also known as dielectric constant. Thus, $\varepsilon_{r}$ for a given material could simply be determined by measuring the capacitance of a capacitor with the material between its plates. The ratio between capacitance with the material $\left(C_{m}\right)$ and capacitance without the material $\left(C_{o}\right)$ gives $\varepsilon_{r}$ according to

$$
\varepsilon_{r}=C_{m} / C_{o}
$$

Meanwhile, conductivity $\sigma$ can be determined through the measurement of dissipation $(D)$ or the loss tangent (the ratio between the imaginary and the real parts of equation (4)) defined as

$$
D=\tan \delta=\sigma /\left(\omega \varepsilon_{o} \varepsilon_{r}\right)=\sigma /(\omega \varepsilon)
$$

Dissipation $D$ can be measured in various ways depending on the measuring instrument, one of which uses measurement of impedance or admittance [17]. This method, proposed by Nelson [17], uses dielectric probes to measure dielectric constant in agricultural grains which physically similar to loose rocks or sands. Using an impedance analyzer or a $\mathrm{Q}$ meter, $D$ could be determined from the following expression [17]

$$
D=\tan \delta=\left(C_{m} D_{m}-C_{o} D_{o}\right) /\left(C_{m}-C_{o}\right)
$$

where $D_{m}$ is the dissipation factor for filled sample holder and $D_{o}$ is the dissipation factor for empty sample holder. 


\section{Data Acquisition and Data Analysis}

The survey is carried out in the village of Bayuran about $20 \mathrm{~km}$ in northeast direction from the town of Jepara, Central Java (see Figure 1). Like any other coast in the vicinity, the iron sand of Bayuran is originated from the old volcano of Muria. This coastal deposit consists of loose silty sands with dark gray color. In many spots, it forms little dunes. Geological map of the area [18] shows no evidence of fault in the vicinity of survey site. Iron sand samples were collected in several randomly selected spots.

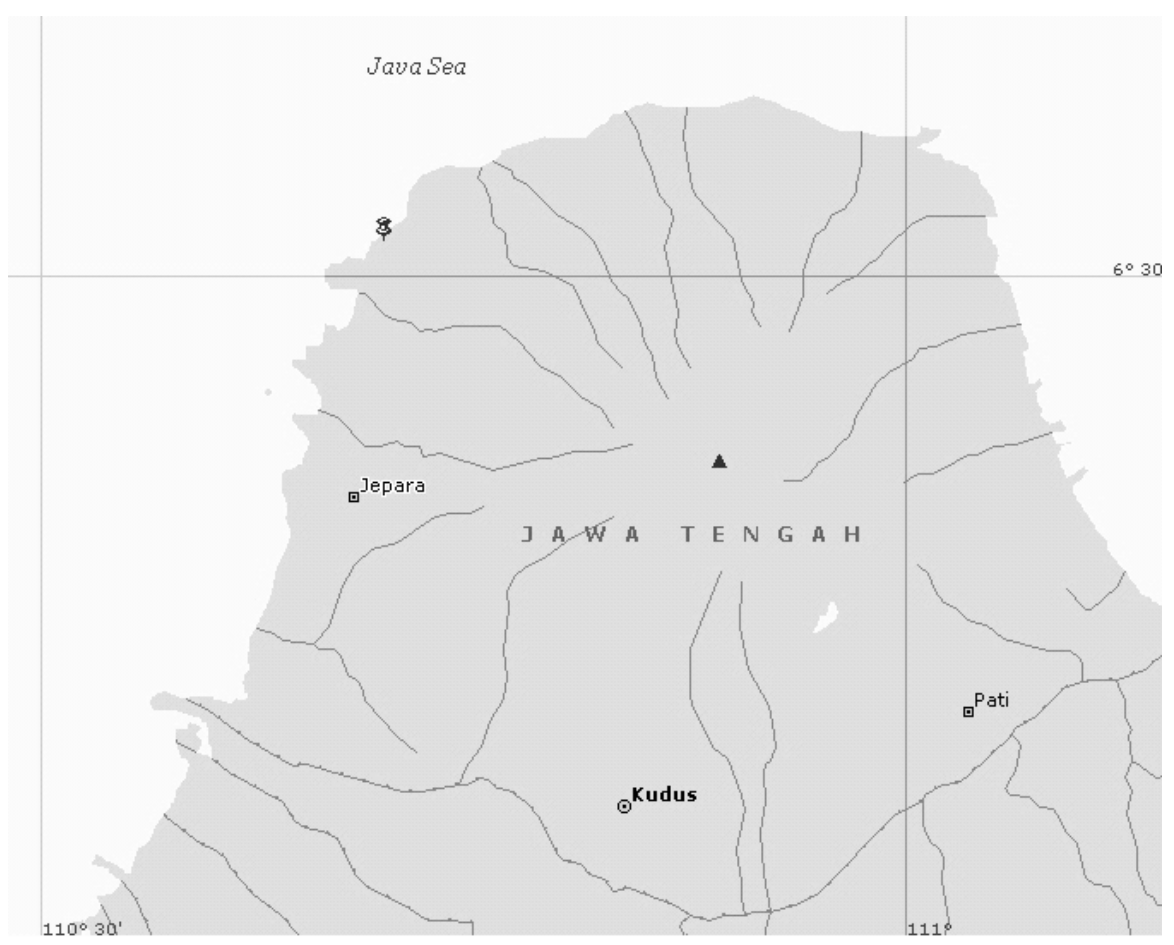

Figure 1 Location of village of Bayuran (push-pin symbol) near the town of Jepara in Central Java. The main feature of the region is Mount Muria (solid triangle).

In the laboratory, the iron sand samples were then subjected to magnetic susceptibility measurement using a Bartington MS2 susceptibility meter (Bartington Instruments Ltd., UK) with an MS2B sensor. The instrument uses a low magnetic field of $80 \mathrm{~A} / \mathrm{m}$ and a frequency of $465 \mathrm{~Hz}$. Sample was contained in cylindrical plastic holder $(2.54 \mathrm{~cm}$ in diameter and $2.2 \mathrm{~cm}$ in length). Mass susceptibilities were measured and later were converted to dimensionless volume susceptibilities $\chi$ as required by equation (3) after the density of the samples had been measured. 
Measurements of relative permittivity $\left(\varepsilon_{r}\right)$ and dissipation factor (D) were carried out first using an HP 4192 LF Impedance Analyzer (Hewlett Packard, USA) with measuring frequency of $200 \mathrm{kHz}$ to $1 \mathrm{MHz}$ at the Department of Engineering Physics at ITB. A specially designed sample holder made of silver plates $(1.8 \mathrm{~cm}$ in diameter) was used to hold the iron sand samples (see Figure 2). Parameters $C_{o}$ and $D_{o}$ were measured when the sample holder is empty while parameters $C_{m}$ and $D_{m}$ were measured when the sample holder is filled with iron sand samples. Measurements were made both for dry sand samples and for wet sand samples. The wet samples, made from sand soaked with seawater, were used to simulate the natural condition of iron sand at the edge of the sea. As working range of the above impedance analyzer is limited to $1 \mathrm{MHz}$, similar measurements were also made with HP Q Meter 4342A (Hewlett Packard, USA). Measurements of relative permittivity $\left(\varepsilon_{r}\right)$ and dissipation factor $(D)$ were also carried out with Q Meter for 10 and $70 \mathrm{MHz}$ frequencies at the Laboratory for Radio Telecommunication and Micro Waves at the Department of Electrical Engineering at ITB.

Back in the field, GPR data were acquired along four lines; two lines were parallel to the coast in East-West direction (lines 26 and 30) and the other two lines were perpendicular to the coast in North-South direction (lines 65 and 66). Lines 26 and 30 are $40 \mathrm{~m}$ long and only a few meters from the water line, whereas lines 65 and 66 are $50 \mathrm{~m}$ long and about $100 \mathrm{~m}$ from the water line. Lines 65 and 66 are grassy with short bushes. Between each pair of survey lines, we placed a point for CMP sounding. There two CMP points in this survey: CMP point 18 and CMP point 33. The schematic of survey lines is given in Figure 3.

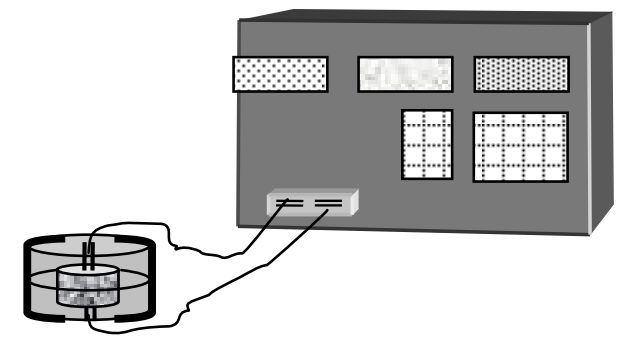

Figure 2 Schematic of sample holder and the HP 4192 LF Impedance Analyzer used in this research.

A pulse radar system with $100 \mathrm{MHz}$ antennas (MALÁ Geoscience, RAMAC GPR) was used in this survey. The $100 \mathrm{MHz}$ frequency was used so that a 
relatively deep region of about 2-15 m can be surveyed. During the reflection profiling, the antennae were separated at a distance of $1 \mathrm{~m}$ and were moved simultaneously over the survey lines. Data we gathered and stored in a notebook computer carried by the operator. For CMP sounding, the transmitting and receiving antennae were placed symmetrically about the CMP point and were moved simultaneously keeping the CMP point as a midpoint between them.

Coast line

\section{Line $26-$ CMP Point 33 \\ Line 30}

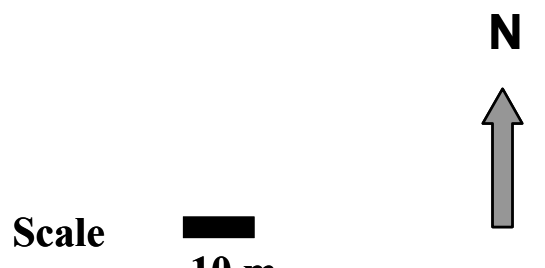

Line 66

Line 65

$\mid$

CMP Point 18

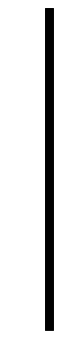

Figure 3 Figure 3 Schematic of survey lines and CMP Points mentioned in this paper.

All the GPR data were processed by specific software termed REFLEXW (Sandmeier Scientific Software, Germany). After exporting the data from RAMAC's format into REFLEXW's format, the process started with filtering that include eliminating low frequency noise due to electronic instrument, 
increasing the amplitude so that deep reflector can still be seen, and applying band pass filter (10-200 MHz) so that the noise is damped. An $f-k$ filtering is also applied to remove small wave number components. Last, we did a deconvolution process to eliminate the effect of multiples on radargram.

After the deconvolution process, the CMP and the reflection profiling data were then processed using separately as each requires different workflow. Once the radargram is acceptable, the CMP data were subjected to CMP-semblance analysis that produces the RMS velocity in velocity-time spectra. The profiling data, meanwhile, were transformed into a time scaled radargram. The time to depth conversion is carried out by entering the appropriate EM velocity of the material.

\section{$4 \quad$ Results and Discussion}

Magnetic susceptibility measurement of several iron sand samples gives an average $\chi$ of 0.308 SI. According to equation (3), this gives $\mu_{r}$ of 1.308 . Meanwhile, measurement of $C_{o}, D_{o}, C_{m}$, and $D_{m}$ for various frequencies from $200 \mathrm{kHz}$ to $1 \mathrm{MHz}$ produced relative permittivity $\left(\varepsilon_{r}\right)$ and dissipation factor $(D)$ shown in Table 1. These values of $\varepsilon_{r}$ and $D$, in turn, were used to calculate $\sigma$ and $V_{m}$ using equations (6) and (2) respectively. As expected, compared to that of wet samples, $\varepsilon_{r}$ is slightly lower for dry samples while $V_{m}$ is higher. The values of $\varepsilon_{r}$ and $V_{m}$ for dry sample are within the range of the published data in the literature [11], i. e., 3-6 for $\varepsilon_{r}$ and 95-170 $\mathrm{mm} / \mathrm{ns}$ for $V_{m}$. However, the values of $\varepsilon_{r}$ and $V_{m}$ for wet sample are out of the range of published data [11](25-30 for $\varepsilon_{r}$ and $55-60 \mathrm{~mm} / \mathrm{ns}$ for $V_{m}$ range). This discrepancy for wet sample may arise from the property of the fluid (sea water in this study versus plain water in the published data). Confirmation of this discrepancy requires further study.

Measurements using $\mathrm{Q}$ meter, meanwhile, shows unreliable results as the meter is very prone to noise. Measurements of $C_{o}, D_{o}, C_{m}$, and $D_{m}$ were carried out only for dry sample. They fluctuate and produce unreliable values of relative permittivity $\left(\varepsilon_{r}\right)$ and dissipation factor $(D)$. Measurement at $70 \mathrm{MHz}$, in particular, was so noisy that the dissipation factor $D$ is out of range. Thus, the $\mathrm{Q}$ meter is deemed to be inappropriate for our purpose.

Meanwhile, Figure 4 shows the typical result of CMP-semblance analysis. This result for CMP point 18 shows that velocity decreases with depth. Event reflectors show interval velocity of $0.153 \mathrm{~m} / \mathrm{ns}$ for the top $0.3 \mathrm{~m}$ followed by an interval velocity of $0.135 \mathrm{~m} / \mathrm{ns}$ for the estimated depth of 0.3 to $3.2 \mathrm{~m}$ and by a velocity of $0.08 \mathrm{~m} / \mathrm{ns}$ for the remaining layer. The first layer is very likely to be topsoil consisting of clays, silts, and sands, while the second layer is the iron sand deposit. 
Table 1 Velocities and other physical parameters measured in the laboratory.

\begin{tabular}{|c|cccc|cccc|}
\hline & \multicolumn{3}{|c|}{ Dry Samples } & \multicolumn{4}{c|}{ Wet Samples } \\
\cline { 2 - 9 }$f$ & $\varepsilon_{r}$ & $D$ & $\sigma$ & $V_{m}$ & $\varepsilon_{r}$ & $D$ & $\Sigma$ & $V_{m}$ \\
$(\mathrm{kHz})$ & & & $\left(10^{-4} \mathrm{~S} / \mathrm{m}\right)$ & $(\mathrm{mm} / \mathrm{ns})$ & & & $\left(10^{-4} \mathrm{~S} / \mathrm{m}\right)$ & $(\mathrm{mm} / \mathrm{ns})$ \\
\hline 200 & 15.80 & 0.99 & 1.73 & 60.18 & 15.80 & 0.91 & 1.59 & 60.88 \\
300 & 9.90 & 1.01 & 1.68 & 75.71 & 11.54 & 0.90 & 1.74 & 71.29 \\
400 & 8.40 & 0.88 & 1.65 & 83.76 & 8.28 & 0.72 & 1.33 & 86.26 \\
500 & 6.37 & 0.75 & 1.32 & 98.07 & 7.66 & 0.65 & 1.38 & 90.53 \\
600 & 5.83 & 0.77 & 1.50 & 102.11 & 6.50 & 0.67 & 1.46 & 98.01 \\
700 & 4.74 & 0.89 & 1.65 & 111.41 & 5.73 & 0.72 & 1.62 & 103.63 \\
800 & 4.18 & 1.16 & 2.16 & 114.10 & 5.19 & 0.93 & 2.15 & 105.88 \\
900 & 3.88 & 1.18 & 2.30 & 117.97 & 4.53 & 0.92 & 2.09 & 113.52 \\
1000 & 3.39 & 1.24 & 2.34 & 125.10 & 4.31 & 0.95 & 2.27 & 115.94 \\
$10 \mathrm{MHz}$ & 4.00 & 0.10 & 2.23 & 130.99 & & & & \\
$70 \mathrm{MHz}$ & 5.09 & 4.71 & 932 & 68.24 & & & & \\
\hline
\end{tabular}

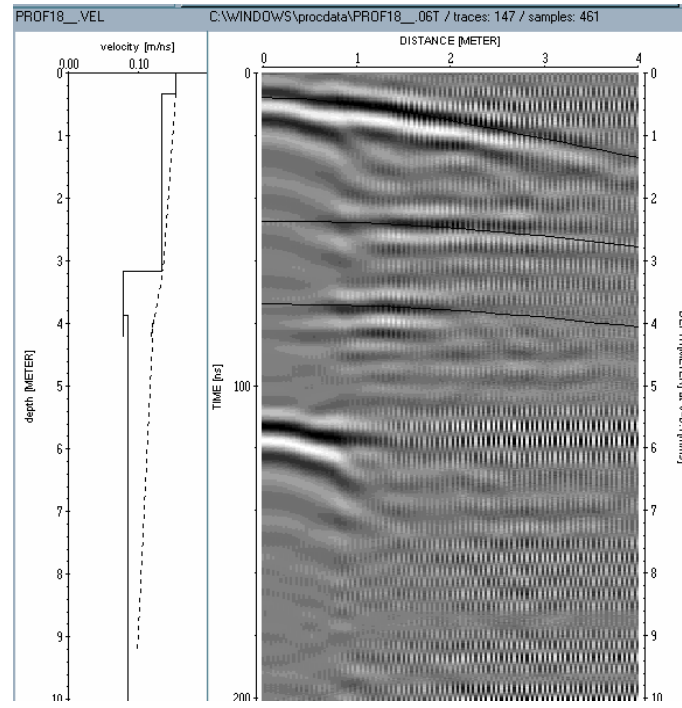

(a)

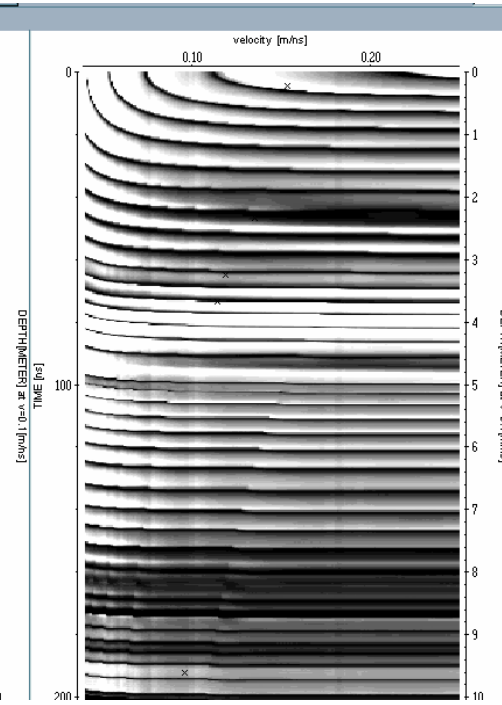

(c)

Figure 4 CMP-semblance analysis for CMP point 18 with (a) interval velocity curve and the estimation of RMS velocity (broken line), (b) results of CMP processing, and (c) the semblance diagram. 

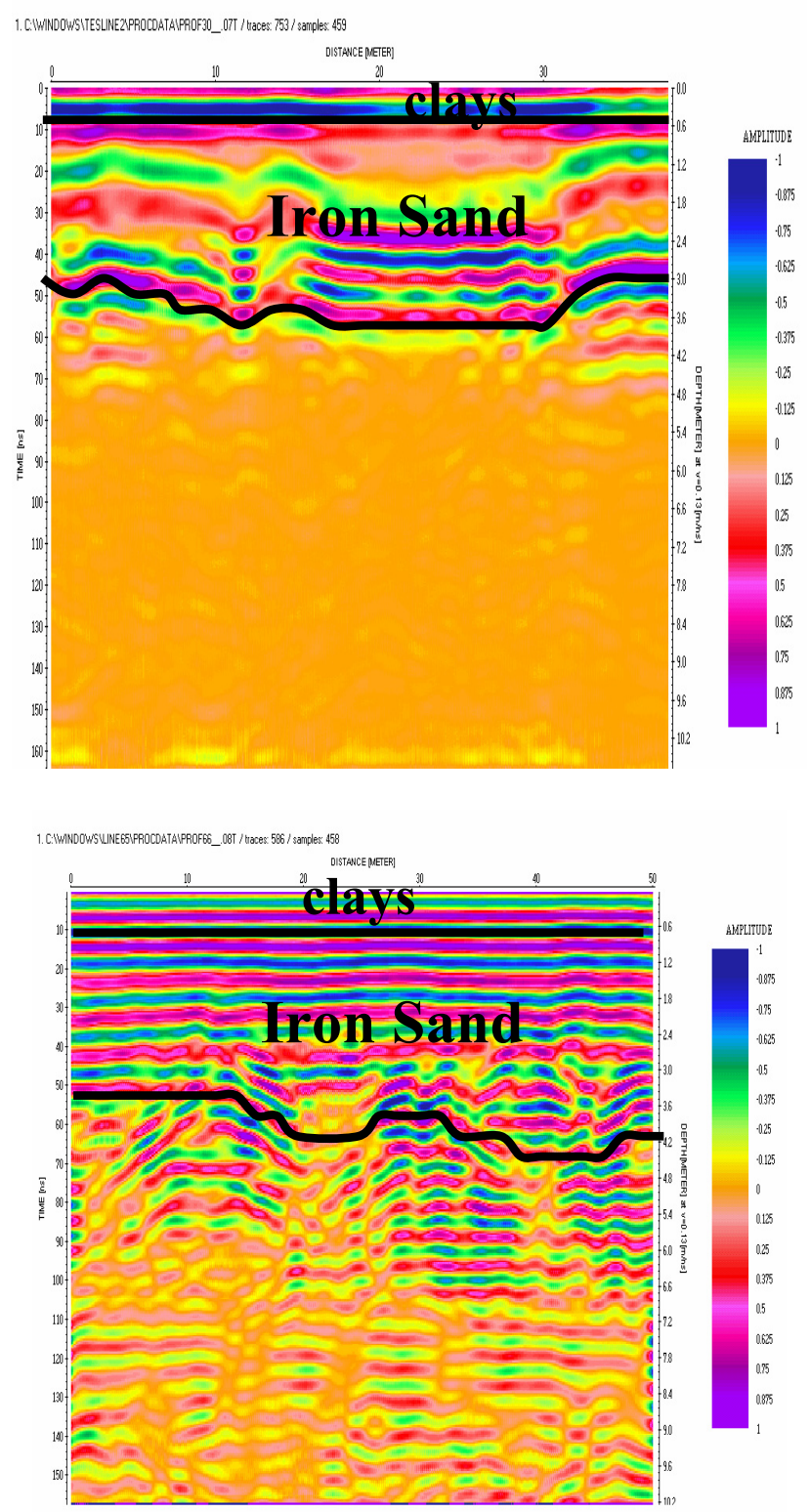

Figure 5 Depth converted GPR profiles of Line 30 (top) and Line 66 (bottom). Solid black lines indicate the boundaries of the iron sand deposit.

The velocities of radiowaves estimated from both laboratory measurement and CMP analysis show considerable match $(125 \mathrm{~mm} / \mathrm{ns}$ from laboratory measurement at frequency of $1 \mathrm{MHz} v \mathrm{vs} 135 \mathrm{~mm} / \mathrm{ns}$ from CMP analysis) despite the fact that the laboratory measurement was carried out in a frequency that is 
lower than the frequency of GPR (1 MHz instead of $100 \mathrm{MHz}$, disregarding the results from $\mathrm{Q}$ meter measurement).

The estimated velocity of radiowaves from CMP analysis was then used as an input for depth conversion in the reflection profiling. Figure 5 shows the depth converted GPR profiles for Line 30 and Line 66. The boundary between iron sand deposit and the surrounding rocks are clearly visible in the profiles. Thus, the depth of the iron sand deposit can be clearly determined. The volume of the deposit can then estimated for economic evaluation.

\section{Conclusion}

The GPR method has been shown to be a reliable method in detecting the vertical extent of iron sand deposit in the village of Bayuran near the town of Jepara in Central Java. Combination of reflection profiling and CMP sounding was deployed for the highly conductive iron sand. Later, CMP-semblance analysis produced the RMS velocity in velocity-time spectra. The interval velocity of iron sand is found to be about $0.135 \mathrm{~m} / \mathrm{ns}$ or $135 \mathrm{~mm} / \mathrm{ns}$. This velocity is then used to convert the profiling data into a depth scaled radargram that shows clearly the boundary of iron sand deposit.

A novel design of the laboratory measurement of EM wave velocity was also tested and applied for iron sand sample. Combining the measurements of magnetic volume susceptibilities $\chi$ and of relative permittivity $\left(\varepsilon_{r}\right)$ and dissipation factor (D), the values of radiowaves velocity, $V_{m}$ were determined as a function of frequency. Results show that HP 4192 LF Impedance Analyzer is better suited for the purpose compared to HP Q Meter 4342A. The Q meter is found to be noisy. The velocities of radiowaves estimated from laboratory match that estimated from CMP analysis.

\section{Acknowledgement}

This study is financially funded by the Hibah Bersaing Research Grant to the senior author (SB). We thank Agus Yulianto and La Ode Ngkoimani for their assistance during the fieldwork. Thanks are also due to Bambang Sunendar and M. Ridwan Effendi for allowing us to use the impedance analyzer at their respective laboratories. Comments and criticism by Wahyudi Parnadi and an anonymous reviewer significantly improve the clarity and readability of this paper. 


\section{References}

1. Harsodo, Perkembangan dan Prospek Pasir Besi Indonesia 1980-1995, Report of PPTM (Pusat Penelitian Teknologi Mineral) Bandung (1991).

2. Yulianto, A., Bijaksana, S. \& Loeksmanto, W., Karakterisasi Magnetik dari Pasir Besi Cilacap, Jurnal Fisika Himpunan Fisika Indonesia A5, 0527.1- 0527.4 (2002).

3. Yulianto, A., Bijaksana, S. \& Loeksmanto, W., Comparative study on magnetic characterization of iron sand from several locations in Central Java, Indonesian Journal of Physics, 14, 63-33 (2003).

4. Yulianto, A., Bijaksana, S., Loeksmanto, W. \& Kurnia, D., Produksi hematit $\left(\mathrm{\alpha Fe}_{2} \mathrm{O}_{3}\right)$ dari pasir besi: Pemanfaatan potensi alam sebagai bahan industri berbasis sifat kemagnetan, Jurnal Sains Materi Indonesia, 5, 51-54 (2003).

5. Davis, J. L. \& Annan, A. P., Ground-penetrating radar for highresolution mapping of soil and rock stratigraphy, Geophysical Prospecting, 37, 531-551 (1989).

6. Goodman, D., Ground-penetrating radar simulation in engineering and archeology, Geophysics, 59, 229-232 (1994).

7. Bohidar, R. N. \& Hermance, J. F., The GPR refraction method, Geophysics, 67, 1474-1485 (2002).

8. Grasmueck, M., Weger, R. \& Horstmeyer, H., Full-resolution $3 D$ GPR imaging, Geophysics, 70, K12-K19 (2005).

9. Xiong, Z. \& Tripp, A. C., Ground-penetrating radar responses of dispersive models, Geophysics, 62, 1127-1131 (1997).

10. Van Dam, R. L., Schlager, W., Dekkers, M. J. \& Huisman, J. A., Iron oxides as a cause of GPR reflections, Geophysics, 67, 536-545 (2002).

11. Reynolds, J. M., An Introduction to Applied and Environmental Geophysics, John Wiley \& Sons (1997).

12. Telford, W. M., Geldart, L. P. \& Sheriff, R. R., Applied Geophysics, $\mathbf{2}^{\text {nd }}$ ed., Cambridge University Press (1990).

13. Schön, J. H., Physical Properties of Rocks: Fundamentals and Principles of Petrophysics, $\mathbf{2}^{\text {nd }}$ ed., Pergamon (1998).

14. Gueguen, Y., \& Palciaukas, V., Introduction to Physics of Rock, Princeton University Press (1994).

15. Greaves, R., Lesmes, D., Lee J. M. \& Toksoz, M. N., Velocity variation and water content estimated from multi-offset, ground penetrating radar, Geophysics, 61, 683-695 (1996).

16. Knight, R. J., Nur, A. \& Raistrick, I. D., Modeling the electrical response of sandstones with an equivalent circuit, SPWLA 26th Annual Logging Symposium, paper M, 1-15 (1985). 
17. Nelson, S. O., Electrical Properties of Agricultural Product (A Critical Review), Transaction of the American of Society Agricultural Engineers (ASAE), 16, 384-400 (1973).

18. Suwarti, T., \& Wikarno, R., Geologi Lembar Kudus, Jawa, Pusat Penelitian dan Pengembangan Geologi Bandung (1992). 